\title{
Feasibility study of one axis three positions tracking solar PV with low concentration ratio reflector
}

\author{
B.J. Huang *, F.S. Sun \\ Department of Mechanical Engineering, National Taiwan University, Taipei 106, Taiwan, ROC
}

Received 20 March 2006; accepted 27 September 2006

Available online 29 November 2006

\begin{abstract}
A new PV design, called "one axis three position sun tracking PV module", with low concentration ratio reflector was proposed in the present study. Every PV module is designed with a low concentration ratio reflector and is mounted on an individual sun tracking frame. The one axis tracking mechanism adjusts the PV position only at three fixed angles (three position tracking): morning, noon and afternoon. This "one axis three position sun tracking PV module" can be designed in a simple structure with low cost. A design analysis was performed in the present study. The analytical results show that the optimal stopping angle $\beta$ in the morning or afternoon is about $50^{\circ}$ from the solar noon position and the optimal switching angle that controls the best time for changing the attitude of the PV module is half of the stopping angle, i.e. $\theta_{\mathrm{H}}=\beta / 2$, and both are independent of the latitude. The power generation increases by approximately $24.5 \%$ as compared to a fixed PV module for latitude $\phi<50^{\circ}$. The analysis also shows that the effect of installation misalignment away from the true south direction is negligible $(<2 \%)$ if the alignment error is less than $15^{\circ}$. An experiment performed in the present study indicates that the $\mathrm{PV}$ power generation can increase by about $23 \%$ using low concentration $(2 X)$ reflectors. Hence, combining with the power output increase of $24.5 \%$, by using one axis three position tracking, the total increase in power generation is about $56 \%$. The economic analysis shows that the price reduction is between $20 \%$ and $30 \%$ for the various market prices of flat plate PV modules.
\end{abstract}

(c) 2006 Elsevier Ltd. All rights reserved.

Keywords: Solar photovoltaic; Solar power; Tracking PV; Concentrator PV

\section{Introduction}

The electricity generation cost of solar PV (photovoltaic) systems is still very high, several times that of conventional power generation. This makes the dissemination of solar PV very difficult.

The tracking flat PV system is one of the methods to reduce the power generation cost. Neville [11] has shown theoretically that for a mid latitude region $\left(\phi=30^{\circ}\right)$, compared to a fixed PV module tilted at an angle equal to the local latitude, the power generation can increase about $41 \%$ using two axis tracking. For a one axis tracking PV system, the power increase is $36 \%$. Several approaches to track the sun using a one axis open loop [6], two axis closed loop [10]

\footnotetext{
* Corresponding author. Tel.: +8862 23634790; fax: +886223640549. E-mail address: bjhuang@seed.net.tw (B.J. Huang).
}

and two axis open loop [12] tracking have been proposed by many researchers. There are also many different controllers to implement the control schemes, e.g. PLA [1] (programmable logic array), PC [14] and microprocessor [7].

Another solution toward cost reduction [3] is to use reflector or Fresnel lens [4] to concentrate the solar radiation incident upon a high efficiency solar cell [13]. It has been shown experimentally that the concentrator PV produces $37 \%$ more energy than the flat plate module in a one year comparison in Northern California. The area of $\mathrm{PV}$ can be largely reduced if the concentration ratio is high $(>100 X)$. However, this requires a high precision two axis sun tracking technology, which is very sophisticated, expensive and less reliable. In the present study, we propose a new idea of one axis three position tracking $\mathrm{PV}$ module with low concentration ratio reflector to provide a more simple PV tracking system, which can reduce the power generation cost. 


\section{Nomenclature}

$A \quad$ altitude of observer, kilometers

$a_{0}, a_{1}, k, a_{0}^{*}, a_{1}^{*}, k^{*}, r_{0}, r_{1}, r_{k}$ parameters for $\tau_{\mathrm{b}}$, dimensionless

$G_{0} \quad$ extraterrestrial (AM0) solar radiation on horizontal plane, $\mathrm{W} / \mathrm{m}^{2}$

$G_{\mathrm{b} n} \quad$ beam radiation on horizontal plane, $\mathrm{W} / \mathrm{m}^{2}$

$G_{\text {sc }} \quad$ solar constant, $1353 \mathrm{~W} / \mathrm{m}^{2}$

$H$ height of shading plate, $\mathrm{mm}$

$I_{\mathrm{c}} \quad$ clear sky horizontal radiation, $\mathrm{J} / \mathrm{m}^{2}$

$I_{\mathrm{cb}} \quad$ clear sky horizontal beam radiation, $\mathrm{J} / \mathrm{m}^{2}$

$I_{\mathrm{cd}} \quad$ clear sky horizontal diffuse radiation, $\mathrm{J} / \mathrm{m}^{2}$

$I_{0 n} \quad$ extraterrestrial (AM0) solar radiation, $\mathrm{J} / \mathrm{m}^{2}$

$I_{\mathrm{T}} \quad$ solar radiation on tilted plane, $\mathrm{J} / \mathrm{m}^{2}$

$L \quad$ distance between photosensing element and shading plate, $\mathrm{mm}$

$n \quad n$th day of year, day

$P_{\mathrm{v}} \quad$ PV module size, $\mathrm{Wp}$

$Q_{\text {opt }} \quad$ optimal yearly total solar incident radiation, $\mathrm{MJ} / \mathrm{m}^{2}$

$Q_{\text {total }} \quad$ yearly total solar incident radiation, $\mathrm{MJ} / \mathrm{m}^{2}$
$R_{\mathrm{b}} \quad$ ratio of beam radiation on tilted surface to that on horizontal surface, dimensionless

$X \quad$ concentration ratio, times

$\alpha \quad$ tilt angle of tracker, degree

$\beta \quad$ stopping angle of tracker, degree

$\gamma \quad$ installation error of tracker, degree

$\delta \quad$ declination, degree

$\theta \quad$ solar incident angle with respect to PV normal vector, degree

$\theta_{\mathrm{H}} \quad$ switching angle of tracker, degree

$\theta_{\mathrm{z}} \quad$ solar incident zenith angle, degree

$\lambda$ slope of PV surface, dimensionless

$\rho \quad$ diffuse ground reflectance, dimensionless

$\tau_{\mathrm{b}} \quad$ atmosphere transmittance for beam radiation, dimensionless

$\tau_{\mathrm{d}} \quad$ atmosphere transmittance for diffuse radiation, dimensionless

$\phi \quad$ latitude of installation location, degree

$\omega \quad$ solar time hour angle, degree

\section{Design analysis of one axis three position sun tracking PV module}

\subsection{Design of one axis sun tracking PV module}

The conventional one axis sun tracking system requires continuous tracking using feedback or open loop control. The tracker is usually designed in a large scale in order to mount multiple flat PV modules. This makes the structure very heavy, complicated and not easy to install. We propose the idea of a simple one axis sun tracking PV module. Every PV module is mounted on an individual sun tracking frame. The one axis tracking mechanism adjusts the PV position only at three fixed angles (three position tracking): morning, noon and afternoon. We call this "one axis three position sun tracking PV". The tracker is designed in simple structure with low cost.
Fig. 1 is the schematic diagram of the one axis three position tracking mechanism. The mechanism includes a single pole support, a tilt adjustable platform, a PV frame driven by a motor and a solar position sensor. The right side of Fig. 1 shows the tilt angle of the tracker, $\alpha$, at the solar noon position by adjusting the platform.

There are three touch switches mounted on the transmission gear of the frame for signal outputting to the control circuit. The PV frame will stop at the touch of the next switch once it is triggered. The designated location of the switch thus determines the stopping angle. Fig. 2 shows the stop positions of the tracker.

Fig. 2(B) is the back view of the PV module facing toward south at solar noon. Three stopping positions are illustrated in Fig. 2 for morning (A), noon (B) and afternoon (C). The PV module at position B (noon) is facing south, while the PV module at positions $\mathrm{A}$ and $\mathrm{C}$ are facing
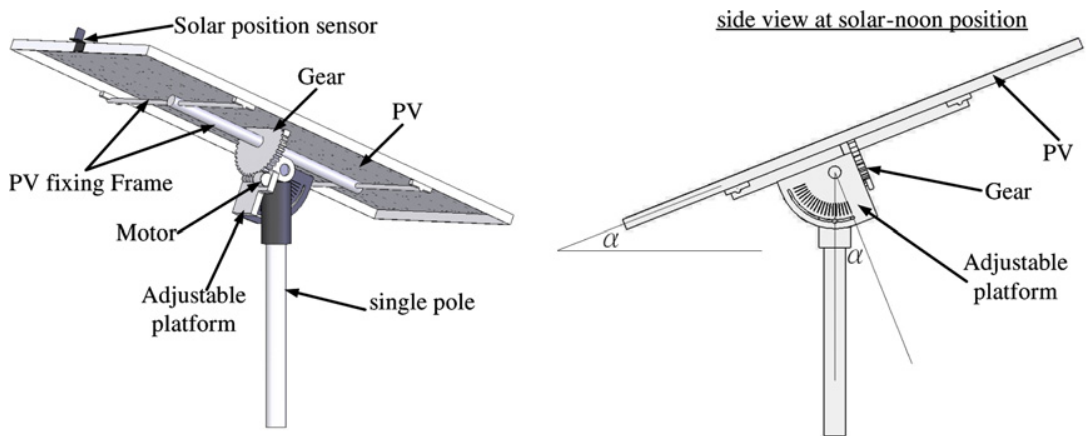

Fig. 1. Schematic diagram of one axis three position tracking PV module. 


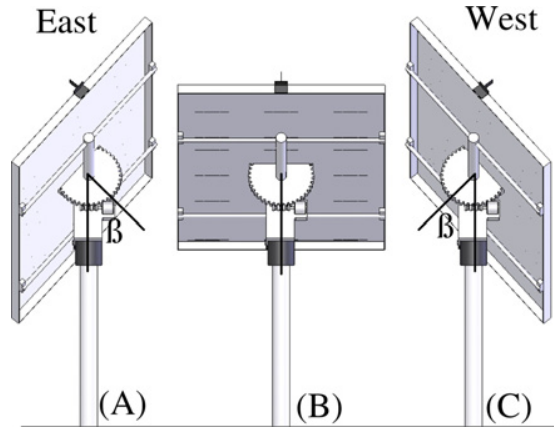

Fig. 2. Concept of one axis three position tracking.

toward the east and west, respectively, with an angle $\beta$ from the noon position.

The sun position sensor consists of two photosensing elements divided by a vertical shading plate (Fig. 3). The sun's motion will finally induce a shadow on one of the photosensing elements. The circuit will detect the signal as well as the relative sun position and actuate the motor to move to the next position, which faces the sun more closely. Since the three position tracking consists of only three stopping angles: morning (east) angle, noon angle and afternoon (west) angle, the control circuit is very simple.

The design of the solar position sensor (Fig. 3) will determine the time for switching the PV to the next position. The height of the shading plate $H$ and the distance of the photosensing element from the plate $L$ will determine the switching time. Once the frame was triggered by the sensor signal, the control circuit will start to move the frame and stop at the next position. This can be achieved through an analog control circuit.

The performance of the one axis three position solar PV system depends on the PV tilt angle $\alpha$ at solar noon, stopping angle $\beta$, installation error $\gamma$ with respect to true south and the switching angle of the sensor $\theta_{\mathrm{H}}$. The switching angle $\theta_{\mathbf{H}}$ is determined by the sensor design following the geometric relation $\tan \theta_{\mathrm{H}}=L / H$.

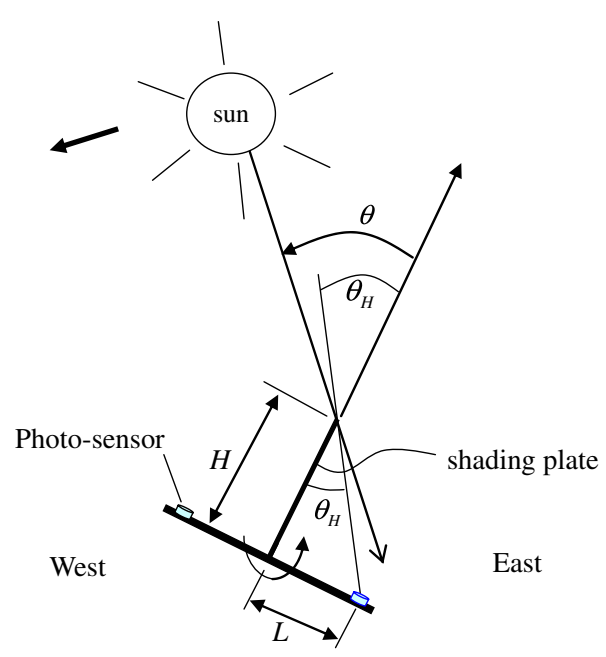

Fig. 3. Schematic of sun position sensor.
The question remaining to be answered is how to find an optimal stopping angle $\beta$ which will generate the highest electric power or receive the maximum incident solar radiation on the PV for a tilt angle $\alpha$ and switching angle of the sensor $\theta_{\mathrm{H}}$ (i.e. sensor design) even with an installation error $\gamma$. A system analysis was first conducted in the present study to determine the yearly total incident solar radiation upon the three position tracking PV system at various design parameters and to find an optimal design.

\subsection{Instantaneous total solar radiation incident upon a one axis three position tracking $P V$}

Referring to Fig. 4 for the coordinate system of the tracking PV, we can calculate the instantaneous solar radiation incident upon the one axis three position tracking PV module using Liu and Jordan's model [9]:

$I_{\mathrm{T}}=I_{\mathrm{cb}} R_{\mathrm{b}}+I_{\mathrm{cd}}\left(\frac{1+\cos \lambda}{2}\right)+I_{\mathrm{c}} \rho\left(\frac{1-\cos \lambda}{2}\right)$,

$R_{\mathrm{b}}=\frac{\cos \theta}{\cos \theta_{\mathrm{z}}}$

where $\rho$ is the ground reflectance, about 0.2 for snow free ground and 0.7 for snow ground, $\lambda$ is the slope of the PV surface; and $I_{\mathrm{cb}}$ is the clear sky horizontal beam radiation, which can be calculated by Hottel's model [5]:

$I_{\mathrm{cb}}=I_{0 n} \tau_{\mathrm{b}} \cos \theta_{\mathrm{z}}$.

The atmosphere transmittance for beam radiation $\tau_{\mathrm{b}}$ is the ratio of the beam radiation $G_{\mathrm{b} n}$ to the air mass zero (AM0) extraterrestrial radiation $G_{0}$,

$\tau_{\mathrm{b}}=G_{\mathrm{b} n} / G_{0}$

that can be expressed as

$\tau_{\mathrm{b}}=a_{0}+a_{1} \mathrm{e}^{-k / \cos \theta_{z}}$.

The constants $a_{0}=r_{0} a_{0}^{*} ; a_{1}=r_{1} a_{1}^{*} ; k=r_{k} k^{*}$ for the standard atmosphere with $23 \mathrm{~km}$ visibility are found from

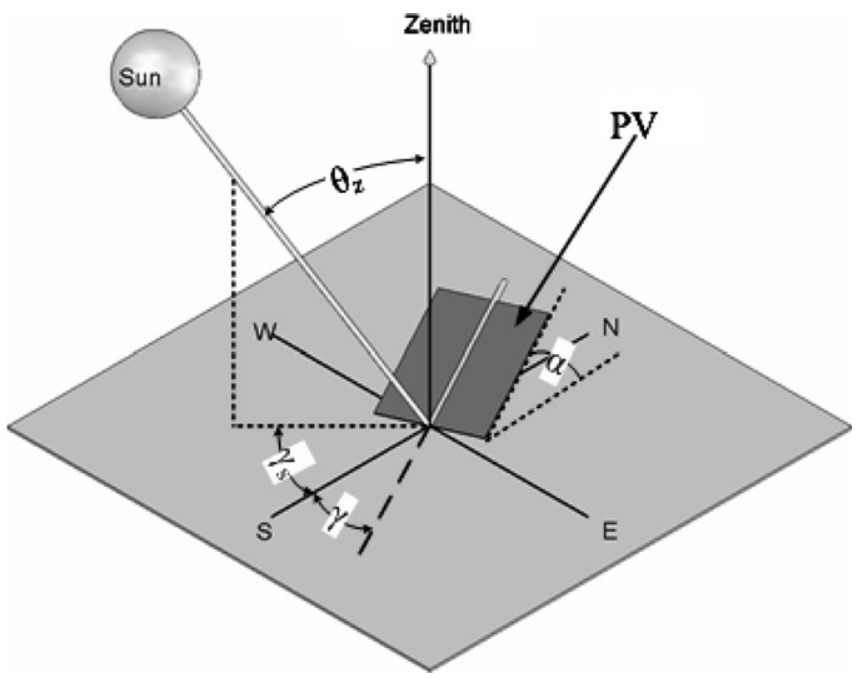

Fig. 4. Geometric definition of the PV module. 
$a_{0}^{*}, a_{1}^{*}$, and $k^{*}$, which are given for altitudes less than $2.5 \mathrm{~km}$ by

$a_{0}^{*}=0.4237-0.00821\left(6-A^{2}\right)$,

$a_{1}^{*}=0.5055+0.00595(6.5-A)^{2}$,

$k^{*}=0.2711+0.01858(2.5-A)^{2}$,

where $A$ is the altitude of the observer in kilometers. The parameters $r_{0}, r_{1}$ and $r_{k}$ depend on the climate type and are given in Table 1 . The variable $I_{0 n}$ is the accumulated extraterrestrial beam radiation (i.e. at AM0) for a period (i.e. $1 \mathrm{~min}$ ) on the $n$th day of the year:

$I_{0 n}=\int G_{0 n} \mathrm{~d} t=\int G_{\mathrm{sc}}\left(1+0.033 \cos \frac{360 \times n}{365}\right) \mathrm{d} t$.

$G_{\text {sc }}$ is the solar constant $\left(1353 \mathrm{~W} / \mathrm{m}^{2}\right)$. The variable $I_{\text {cd }}$ in Eq. (1) is the diffuse radiation, which can be calculated using Liu and Jordan's model [8]:

$I_{\mathrm{cd}}=I_{0 n} \tau_{\mathrm{d}} \cos \theta_{\mathrm{z}}$

$\tau_{\mathrm{d}}=0.2710-0.2939 \tau_{\mathrm{b}}$.

Referring to Fig. 4, the solar incident angle with respect to the PV normal vector $\theta$, and the zenith angle $\theta_{\mathrm{z}}$ are related to the PV installation tilt angle $\alpha$, stopping angle $\beta$ and solar declination angle $\delta$, which can be calculated by Eqs. (10) and (11), which are derived from the relative position of the sun and the PV attitude [2]:

$$
\begin{aligned}
\cos \theta= & \sin \alpha \cos \beta \sin \phi \cos \gamma \cos \delta \cos \omega \\
& -\sin \alpha \cos \beta \cos \gamma \cos \phi \sin \delta \\
& -\sin \beta \sin \gamma \sin \phi \cos \delta \cos \omega+\sin \beta \sin \gamma \cos \phi \sin \delta \\
& +\sin \alpha \cos \beta \sin \gamma \cos \delta \sin \omega+\sin \beta \cos \gamma \cos \delta \sin \omega \\
& +\cos \alpha \cos \beta \sin \phi \sin \delta+\cos \alpha \cos \beta \cos \phi \cos \delta \sin \omega,
\end{aligned}
$$

$\cos \theta_{\mathrm{z}}=\cos \phi \cos \delta \cos \omega+\sin \phi \sin \delta$,

$\cos \lambda$ in Eq. (1) is the inner product of the PV normal vector and the zenith vector, which is given by

$\cos \lambda=\cos \alpha \cos \beta$,

$\delta=23.45 \sin \left[360 \frac{284+n}{365}\right], \quad \operatorname{deg}$,

$\gamma$ is the azimuth or installation deviation (misalignment error) angle of the inclined PV surface away from true south (negative for eastward and positive for westward); $\phi$ is the latitude of the installation location; $\omega$ is the solar time hour angle (negative in the morning, positive in the afternoon and zero at solar noon; 15 degree per hour).

Table 1

$r_{0}, r_{1}$, and $r_{k}$

\begin{tabular}{llll}
\hline Climate type & $r_{0}$ & $r_{1}$ & $r_{k}$ \\
\hline Tropical & 0.95 & 0.98 & 1.02 \\
Mid-latitude summer & 0.97 & 0.99 & 1.02 \\
Sub-arctic summer & 0.99 & 0.99 & 1.01 \\
Mid-latitude winter & 1.03 & 1.01 & 1.00 \\
\hline
\end{tabular}

\subsection{Optimal design of one axis three position tracking $P V$} module

The optimal design of the one axis three position tracking PV module can be made by calculating the total solar radiation incident upon the PV at various design parameters. Given the design parameters, we can calculate the instantaneous total solar radiation incident upon a one axis three position tracking PV module using Eqs. (1)-(13). The yearly total solar incident radiation on the PV surface can then be calculated through time integration.

(1) Optimal stop angle for the design with PV tilt angle equal to location latitude $(\alpha=\phi)$ : Many theoretical studies show that the maximum solar incident radiation can be obtained if the tilted surface angle approximately equals the latitude $(\alpha=\phi)$. Hence, we first calculate the yearly total solar incident radiation $Q_{\text {total }}$ for various designs of stopping angle $\beta$ at a tilt angle $\alpha=25^{\circ}$, which equals the latitude of Taiwan. For each stopping angle, we calculate $Q_{\text {total }}$ at various switching angles of the sensor $\left(\theta_{\mathbf{H}}\right)$ and find the maximum $Q_{\text {total }}$, denoted $Q_{\text {opt }}$. Fig. 5 shows that the optimal stopping angle is $50^{\circ}$, and the optimal switching angle is $25^{\circ}$, which is half of the stopping angle. It can be shown from the results of the calculated $Q_{\text {total }}$ that the $\mathrm{PV}$ power generation will increase by $24.5 \%$ as compared to a fixed PV module.

(2) Optimal tilt angle with $\alpha \neq \phi$ : For a more general case with $\alpha \neq \phi$, we repeat the above calculation for different solar noon tilt angles $\alpha$ at different latitudes $(\phi$ $=25^{\circ}, 45^{\circ}, 65^{\circ}$ ) to determine the optimal tilt angle $\alpha$ and stop angle $\beta$. Fig. 6 presents the optimal tilt angle and optimal stopping angle. Table 2 summarizes the optimal design conditions for various latitudes. It is seen that the optimal stopping angle $\beta$ is about $50^{\circ}$ regardless of the latitude, and the optimal switching angle is half of the stopping angle, i.e. $\theta_{\mathrm{H}}=\beta / 2$, irrespective of the latitude (Table 2 and Fig. 6). This is an important conclusion for the optimal design of the present one axis three position tracking PV module.

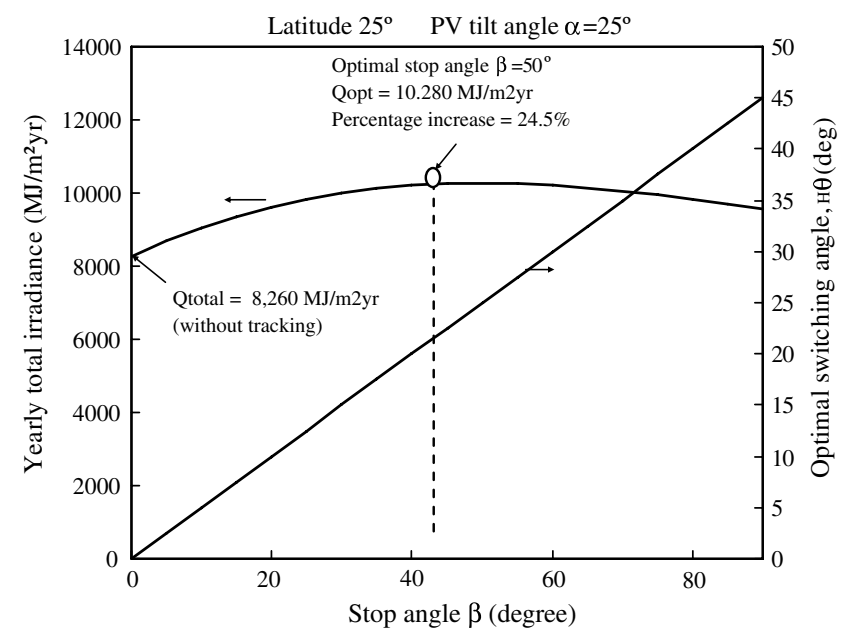

Fig. 5. Variation of yearly total irradiance on PV with the design of stop angle $\beta$. 


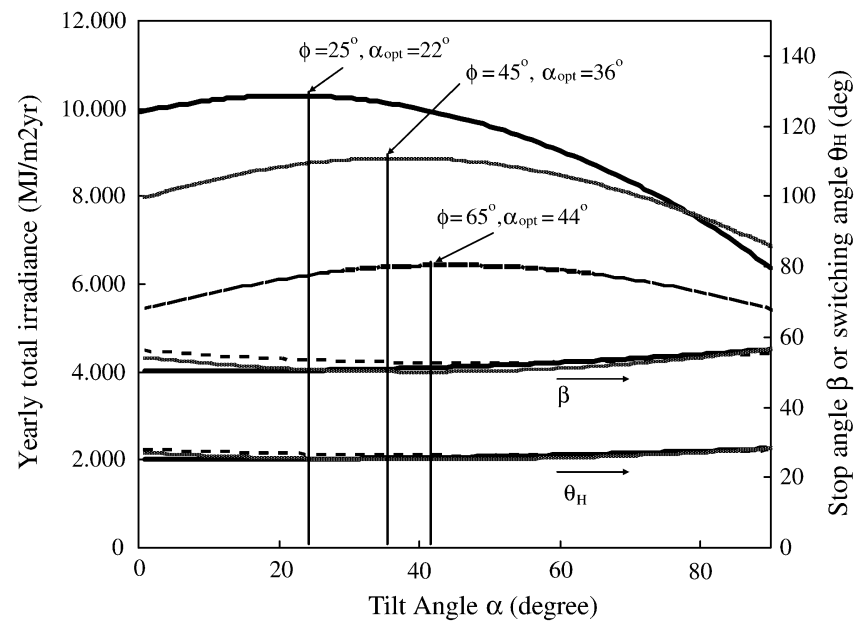

Fig. 6. Variation of yearly total irradiance with tilt angle.

Table 2

Optimal design at different latitude

\begin{tabular}{llll}
\hline & \multicolumn{2}{l}{ Latitude $\phi$} & \\
\cline { 2 - 4 } & $25^{\circ}$ & $45^{\circ}$ & $65^{\circ}$ \\
\hline Optimal tilt angle $\alpha$ & $22^{\circ}$ & $36^{\circ}$ & $44^{\circ}$ \\
Optimal stop angle $\beta$ & $50^{\circ}$ & $50^{\circ}$ & $52^{\circ}$ \\
Optimal switching angle $\theta_{\mathrm{H}}$ & $25^{\circ}$ & $25^{\circ}$ & $26^{\circ}$ \\
$Q_{\text {opt }}\left(\mathrm{MJ} / \mathrm{m}^{2}\right)$ & 10,289 & 8871 & 6429 \\
$Q_{\text {total }}$ no tracking $\left(\mathrm{MJ} / \mathrm{m}^{2}\right)$ & 8263 & 7149 & 5047 \\
Percentage increase $(\%)$ & 24.5 & 24.1 & 27.4 \\
\hline
\end{tabular}

Fig. 7 shows the results for the design following the rule $\theta_{\mathbf{H}}=\beta / 2$. It shows that the power generation increases by approximately $24.5 \%$ as compared to the fixed PV module for latitude $\phi<50^{\circ}$.

(3) Effect of installation error: Usually, the PV module installation cannot perfectly be facing the exact south direction all the time. The power generation will be reduced due to a misalignment away from the true south direction. The total solar radiation incident upon the PV module with the optimal design shown above (i.e. optimal tilt angle, $\beta=50^{\circ}$ and $\theta_{\mathrm{H}}=\beta / 2$ ) can be calculated for different mis-

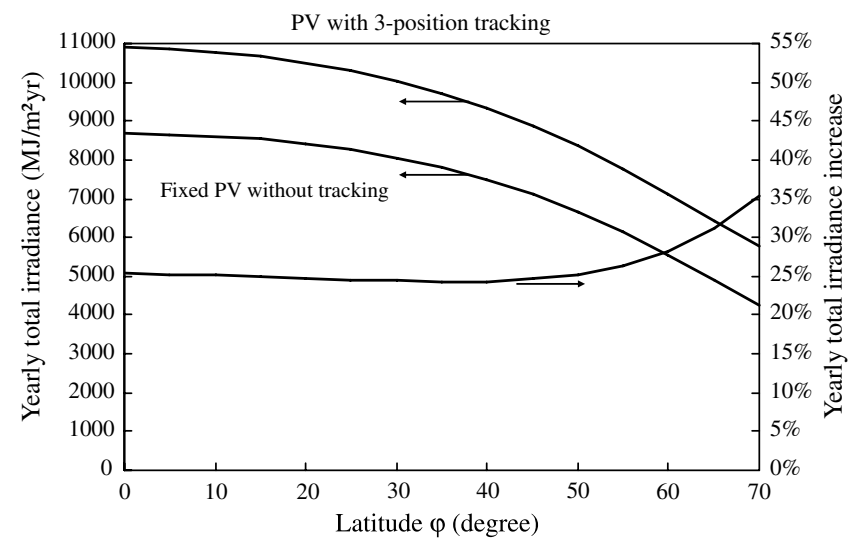

Fig. 7. Yearly total irradiance for the design following the rule $\theta_{\mathbf{H}}=\beta / 2$. alignment angles $\gamma$ at various latitudes $\phi$. The results shown in Figs. 8 and 9 indicate that the effect of misalignment is negligible $(<2 \%)$ if the alignment error is less than $15^{\circ}$. At a mid-low latitude region $\phi<40^{\circ}$, the reduction of power generation due to misalignment is about $5 \%$, even if the alignment error is $35^{\circ}$.

\section{Power generation enhancement of one axis three position sun tracking PV module with low concentration ratio reflector}

In the previous section, the design of the one axis three position tracking PV module is determined by calculating the total solar radiation incident upon the PV surface. In order to increase the power generation, we may further add a simple reflector on the PV module to build a concentrator PV (cPV). A low concentration ratio reflector $(X<3)$ is preferred since it causes less temperature effect for the commercial flat plate PV module. An experiment was conducted in the present study to measure the power generation enhancement of $\mathrm{cPV}$ in use with a $2 X$ concentration ratio reflector.

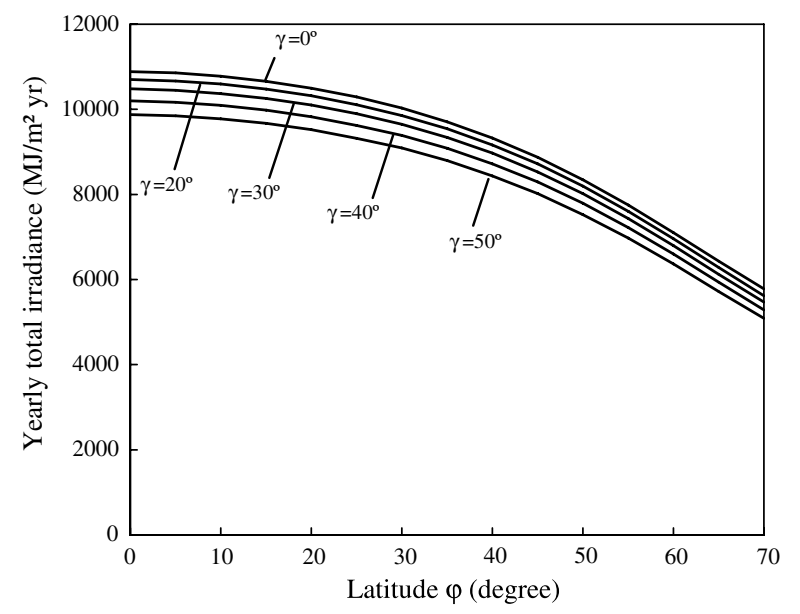

Fig. 8. Variation of yearly total irradiance on PV module with alignment error.

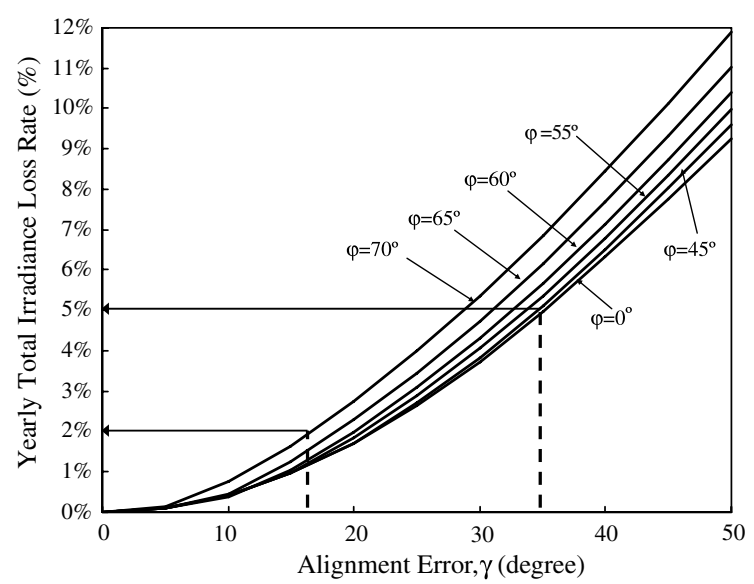

Fig. 9. PV power generation reduction due to alignment error. 
The experiment used an $85 \mathrm{Wp}$ flat plate PV module [FMSN-85W-R02] with 36 solar cells arranged in six parallel arrays. The solar cell is made from single crystal silicon with conversion efficiency of $16 \%$. A $60^{\circ}$ triangular shape reflector is placed between two arrays. The aperture area is $750 \mathrm{~mm} \times 1500 \mathrm{~mm}$, and the total area of solar cells is $750 \mathrm{~mm} \times 750 \mathrm{~mm}$, which means the concentration ratio of this module is 2 (Fig. 10).

To determine the power generation enhancement due to the concentration reflector, we built another test rig using the same PV module but without reflector to measure the power generation simultaneously. Both PV modules were mounted on a fixed frame side by side with tilt angle $25^{\circ}$ facing toward south as shown in Fig. 11. The continuous monitoring data at one day in Fig. 12 shows that the instantaneous PV power generation of cPV with $2 X$ reflector can increase by about $50 \%$ at good condition (strong wind, high irradiation $>600 \mathrm{~W} / \mathrm{m}^{2}$, narrow solar incident angle) and mostly within $20-40 \%$ at high irradiation. The daily total solar power generation increases by $23 \%$ for cPV with $2 X$ reflector as compared to the flat plate PV. The variation of power generation enhancement for $\mathrm{cPV}$ is random due to weather conditions. Together with the test results of many researchers [3,4,13], it is believed that $23 \%$ power generation enhancement is a conservative estimation. We, thus, use this result for evaluating the eco-

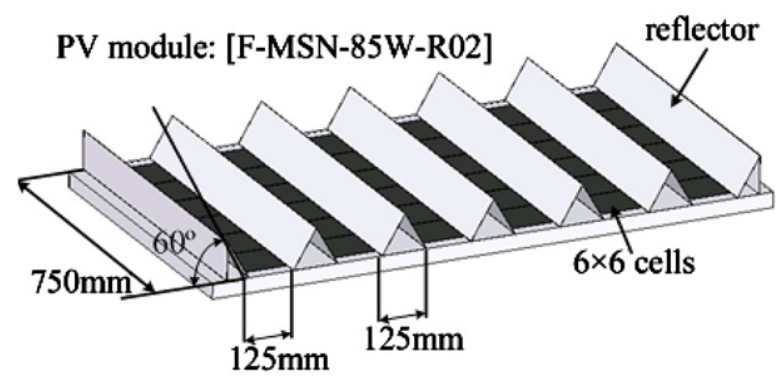

Fig. 10. Schematic diagram of low concentration ratio $(2 X) \mathrm{cPV}$ module.

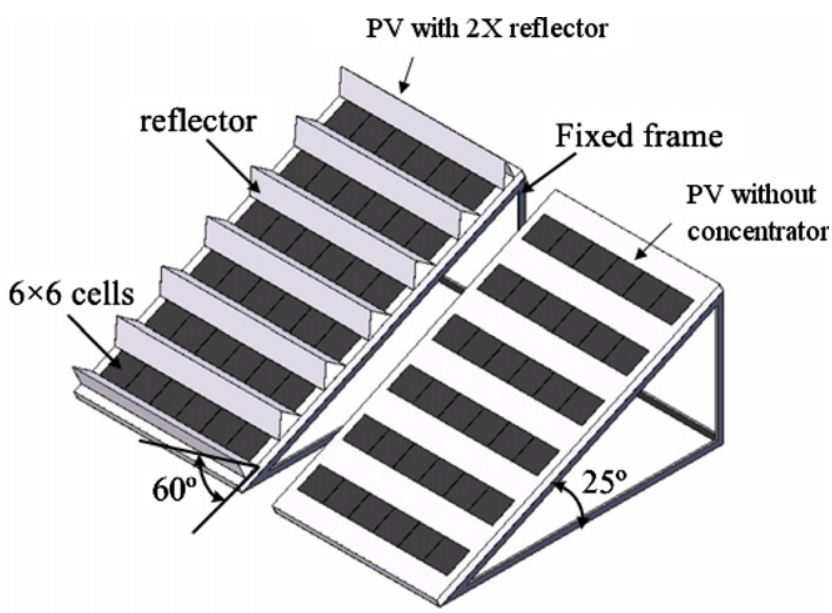

Fig. 11. Schematic diagram of comparison test of two PV modules.

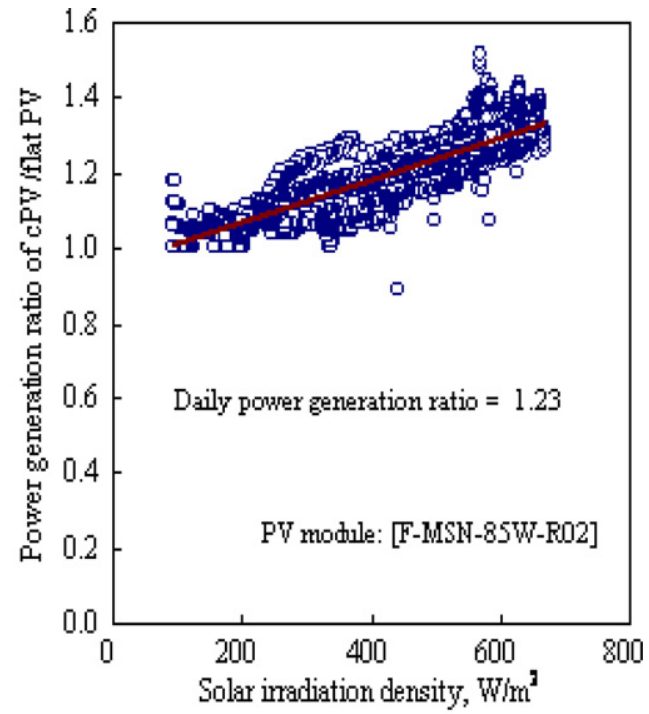

Fig. 12. Variation of power generation ratio with solar irradiation for two PV modules (2004/06/18)

nomic feasibility of a one axis three position sun tracking PV module with low concentration ratio reflector.

\section{Economic feasibility of one axis three position sun tracking PV module with low concentration ratio reflector}

The above test results show that PV power generation can be increased by about $23 \%$ using a low concentration $(2 X)$ reflector. In addition, the previous analysis shows that the one axis three position tracking will increase power output by approximately $24.5 \%$. Combining both, we can have about $56 \%$ increase in power generation. It is estimated that the additional cost for the tracking mechanism, reflector and PV packaging is estimated to be 100 US dollars for a $100 \mathrm{Wp}$ PV module. The additional cost will be higher for larger PV modules due to the increase in structure weight and $\mathrm{PV}$ area. It is estimated the additional cost $C_{\mathrm{a}}$ (in USD) for a PV module size $P_{\mathrm{v}}(\mathrm{Wp})$ follows the following relation:

$C_{\mathrm{a}}=100+0.5\left(P_{\mathrm{v}}-100\right), \quad$ in USD.

For example, for a PV module size $200 \mathrm{Wp}$, the additional cost is 150 USD, which needs to be added to the sale price, but the additional power output of $112 \mathrm{Wp}$ can be created by this new design. That is, the new PV module can be rated as $312 \mathrm{Wp}$. The net value gained is, thus, 410 US dollars based on the present market price of flat plate PV modules, USD 5 per $\mathrm{Wp}$. This corresponds to a price reduction $26.3 \%$ at the sale price of $3.69 \mathrm{USD} / \mathrm{Wp}$ (total price USD 1150 per module). A price reduction of $19.9 \%$ is still possible even if the market price of the PV module is down to USD 3/Wp in the future. Fig. 13 summarizes the new PV price and the price reduction percentage of the present new PV module with one axis three position tracking and $2 X$ concentration reflector. It can be seen from Fig. 13 that the price reduction will not 


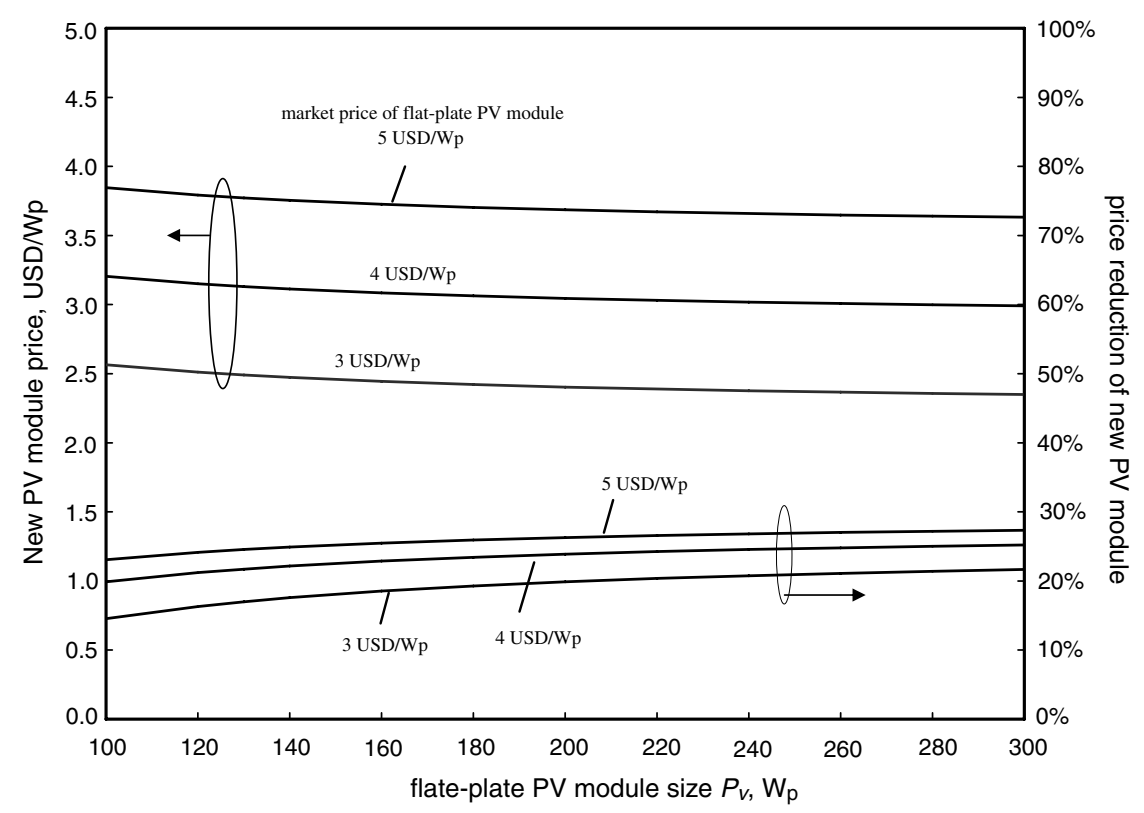

Fig. 13. Price reduction estimation for PV module with one axis three position tracking with $2 X$ concentrator reflector.

change too much if the size of the flat plate PV module is larger than $160 \mathrm{Wp}$. Fig. 13 indicates that the price reduction is always between $20 \%$ and $30 \%$ for various market prices of flat plate PV modules.

For smaller PV module size $(<100 \mathrm{Wp})$, the price reduction of a PV module with one axis three position tracking and $2 X$ concentration reflector becomes not significant if the market price of the flat plate PV module is less than USD 3/Wp.

\section{Discussion and conclusion}

A new design idea of a one axis three position tracking PV module was proposed in the present study. Every PV module is mounted on an individual sun tracking frame. The one axis tracking mechanism adjusts the PV position only at three fixed angles (three position tracking): morning, noon and afternoon.

A design analysis of the one axis three position sun tracking PV module was performed in the present study. The analytical results show that the optimal stopping angle $\beta$ is about $50^{\circ}$, and the optimal switching angle, which controls the best time for changing the attitude of the PV module is half of the stopping angle, i.e. $\theta_{\mathrm{H}}=\beta / 2$, and both values are regardless of the latitude. The power generation increases by approximately $24.5 \%$ as compared to a fixed PV module for latitudes $\phi<50^{\circ}$.

The analysis also indicates that the effect of installation misalignment away from the true south direction on the total solar radiation incident upon the PV module or PV power generation is negligible $(<2 \%)$ if the alignment error is less than $15^{\circ}$. At mid-low latitude regions $\phi<40^{\circ}$, the reduction of power generation due to misalignment is about $5 \%$ even if the alignment error is $35^{\circ}$. The analytical results show that the present one axis three position sun tracking PV module is quite promising in cost reduction. This "one axis three position sun tracking PV module" can be designed in a simple structure with low cost.

The present analysis shows that the PV power generation can be increased further by about $23 \%$ using a low concentration $(2 X)$ reflector in addition to the power output increase of $24.5 \%$ by using one axis three position tracking. Combining both, the total increase in power generation is about $56 \%$.

The economic analysis shows that for a PV module size of $200 \mathrm{Wp}$, the price reduction is $26.3 \%$ at a sale price of $3.69 \mathrm{USD} / \mathrm{Wp}$. A price reduction of $19.9 \%$ is still possible even if the market price of the flat plate PV module is down to USD $3 / \mathrm{Wp}$ in the future. The price reduction will not change too much if the size of the flat plate PV module is larger than $160 \mathrm{Wp}$. For smaller flat plate PV module sizes $(<100 \mathrm{Wp})$, the price reduction for a PV module with one axis three position tracking and $2 X$ concentration reflector becomes not significant if the market price of the flat plate PV module is less than USD $3 / \mathrm{Wp}$.

\section{Acknowledgement}

The present study was supported by the Radistar Co. and partially by the Energy Bureau, Ministry of Economic Affairs, Taiwan.

\section{References}

[1] Abouzeid M. Use of a reluctance stepper motor for solar tracking based on a programmable logic array (PLA) controller. Renew Energy 2001;23:551-60.

[2] Duffie JA, Beckman WA. Solar engineering of thermal processes. New York: Wiley Interscience; 1980. p. 28-110. 
[3] Fraas L, McConnell B. High power density photovoltaics - a path to cost-competitive solar electric power. Renew Energy World 2002;5(5): 99-110.

[4] Garboushian V, Roubideaux D, Yoon S. Integrated high-concentration PV near-term alternative for low-cost large-scale solar electric power. Solar Energy Mater Solar Cells 1997;47(1-4):315-23.

[5] HottelHC.Asimplemodelforestimating thetransmittance of direct solar radiation throughclear atmospheres. SolarEnergy 1976;18(2):129-34.

[6] Kalogirou SA. Design and construction of a one-axis sun-tracking system. Solar Energy 1996;57(6):465-9.

[7] Koyuncu B, Balasubramanian K. A microprocessor controlled automatic sun tracker. IEEE Trans Consum Electron 1991;37(4): 913-917.

[8] Liu BYH, Jordan RC. The interrelationship and characteristic distribution of direct, diffuse and total solar radiation. Solar Energy 1960;4(3):1-19.

[9] Liu BYH, Jordan RC. The long-term average performance of flatplate solar energy collectors. Solar Energy 1963;7(2):53-74.
[10] Lynch WA, Salameh ZM. Simple electro-optically controlled dualaxis sun tracker. Solar Energy 1990;45(2):65-9.

[11] Neville RC. Solar energy collector orientation and tracking mode. Solar Energy 1978;20(1):7-11.

[12] Park K, Lee JH, Kim SH, Kwak YK. Direct tracking control using time-optimal trajectories. Control Eng Practice 1996;4(9): $1231-1240$

[13] Verlinden PJ, Terao A, Daroczi S, Crane RA, Mulligan WP, Cudzinovic MJ, et al. One-year comparison of a concentrator module with silicon point-contact solar cell to a fixed flat plate module in Northern California. In: Proceedings of the 16th European photovoltaic solar energy conference, May 1-5, 2000, Glasgow, UK, 2000. <http://www.sunpowercorp.com/html/ Resources/TP_index.html $>$.

[14] Yousef HA. Design and implementation of a fuzzy logic computercontrolled sun tracking system. Proc IEEE Int Symp Ind Electron 1999;3:1030-4. 